\title{
Remnant of the common pulmonary vein mistaken for a left atrial mass: clarification by transoesophageal echocardiography
}

\author{
Warren J Manning, Carol A Waksmonski, Marilyn F Riley
}

\begin{abstract}
Faulty incorporation of the common pulmonary vein leaves it as a distinct structure posteriorly, into which the pulmonary veins empty. This "chamber" is separated from the anterior "fetal" left atrium (containing the left atrial appendage and communicating with the mitral valve) by a diaphragm, and is known as cor triatriatum, one of the rarest of cardiac malformations. Less pronounced but still incomplete regression of this vein would result in the persistence of a portion of the common pulmonary vein appearing as a mass along the lateral wall of the left atrium at the junction of the left atrial appendage and left upper pulmonary vein. In two patients, both referred for evaluation of a left atrial mass, transoesophageal echocardiography identified the "mass" as a remnant of the common pulmonary vein.

Cardiologists need to be aware of this structural remnant and its possible variants so as to avoid misdiagnosis of this prominence as an atrial tumour or mass.
\end{abstract}

Transthoracic cross sectional echocardiography has revolutionised the antemortem examination of the heart for tumours and thrombi. Limitations of the transthoracic examination, however, include the dependence on transducer position and angulation ${ }^{1}$ and the frequent inability to image adequately the left atrial appendage and pulmonary veins. More recently transoesophageal echocardiography has been shown to provide superior visualisation of the left atrium, left atrial appendage, and pulmonary veins. ${ }^{2}$ We report the cases of two patients who were referred to our hospital for further evaluation of left atrial "tumours" identified by transthoracic echocardiographic study. In both instances, transoesophageal echocardiographic examination showed a prominent confluence of the left atrial appendage and left upper pulmonary vein.

\section{Case reports}

CASE 1

A 60 year old white woman with a history of a remote myocardial infarction, mild aortic stenosis, and mild aortic insufficiency was admitted for evaluation of acute onset rightsided hemiparesis and dysarthria. A computed tomogram of the head without contrast was normal. Transthoracic echocardiography, to exclude a cardiac source of emboli, showed normal chamber sizes and left ventricular systolic function. An elongated left atrial mass was noted originating at the base of the left atrial appendage (fig 1A). She was transferred for further evaluation. Transoesophageal echocardiography showed normal chamber sizes with a prominent confluence of the left atrial appendage and left upper pulmonary vein extending $2.5 \mathrm{~cm}$ into the body of the left

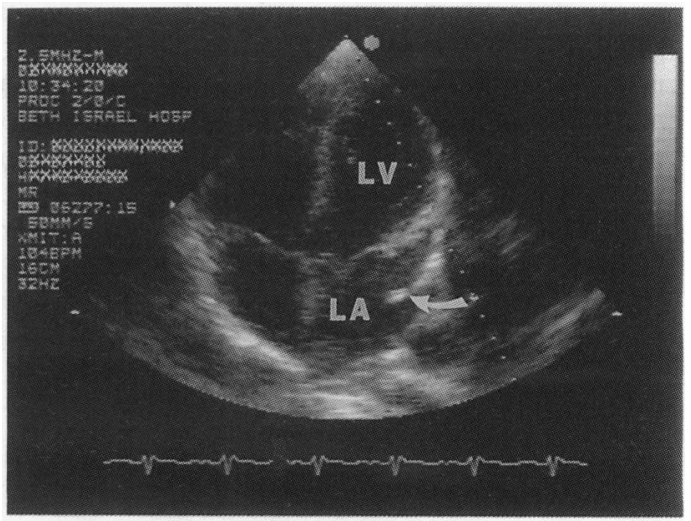

( $A$ )

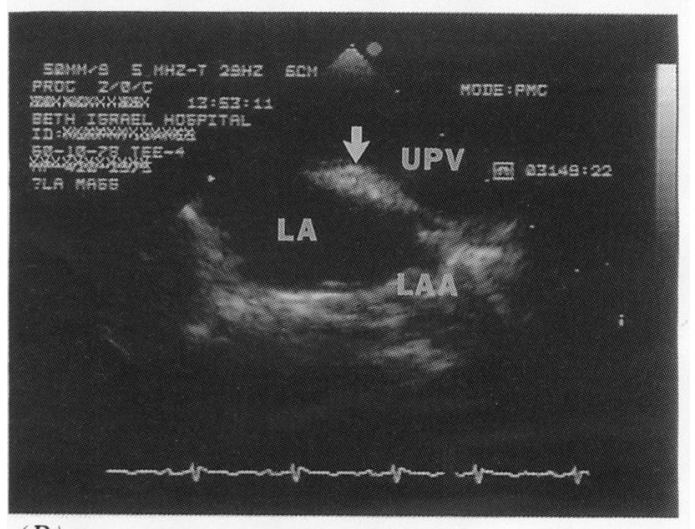

(B)

Figure 1 (A) Apical four chamber view showing an elongated echodensity/mass (arrow) originating near the base of the left atrial appendage. $L A$, left atrium; $L V$, left ventricle. (B) Transoesophageal study of the patient shown in $(A)$, showing prominence (arrow) of the confluence of the left atrial appendage (LAA) and left upper pulmonary vein (UPV). 
Figure 2 Apical four chamber view showing left atrial enlargement and $a$ linear echodensity/mass along the lateral wall (arrow) of the left atrium.

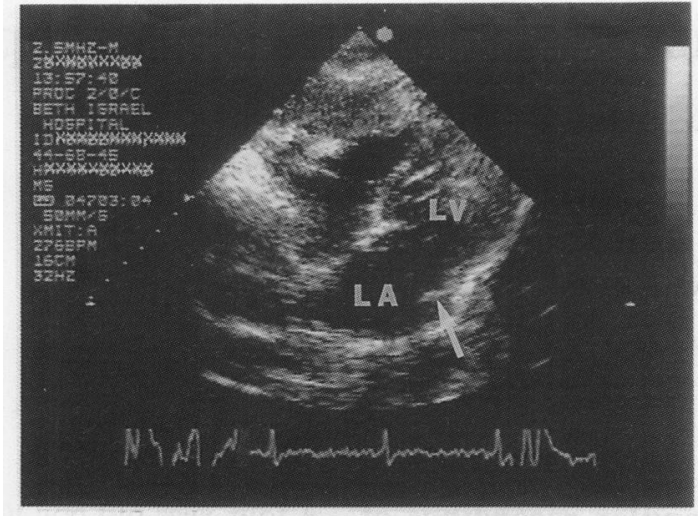

atrium (fig 1B). No evidence of an intracardiac mass was seen.

\section{CASE 2}

A 67 year old white man was well until his vision became blurred a week before admission. Physical examination showed isolated hemianopsia and was otherwise normal. A computed tomogram of the head showed an occipital infarct. Transthoracic echocardiography, performed to exclude a cardiac source of emboli, showed mild left atrial enlargement and a left atrial mass (fig 2). He was referred for further evaluation. transoesophageal echocardiography was performed to define better the abnormality visualised by transthoracic study. It showed an enlarged left atrium with a prominent confluence of the left atrial appendage and left upper pulmonary vein. No evidence of tumour, thrombus, or other mass was seen.

\section{Discussion}

Primary intracardiac tumours are rare: they account for less than $0.3 \%$ of all tumours identified at necropsy. ${ }^{3}$ Most intracardiac tumours are myxomas, of which most arise from the left atrium, with lipomas, papillary fibroelastomas, angiosarcomas, rhabdomyomas, and fibromas being increasingly less common. ${ }^{3}$ Antemortem diagnosis of intracardiac tumours has become common with the development of cardiac ultrasound. Transthoracic cross sectional echocardiography has revolutionised the examination of the heart for tumours and thrombi, because it shows the spatial orientation and accurately defines the location, size, shape, and site of attachment of intracardiac tumours. ${ }^{4}$

The pulmonary veins develop as a posterior protrusion from that portion of the embryonic heart destined to become the left atrium.' This protrusion, the common pulmonary vein, is a temporary structure that communicates with the splanchnic plexus to establish pulmonary venous drainage to the left atrium.

During the fifth embryonic week the common pulmonary vein vanishes as it becomes incorporated in the left atrium proper. ${ }^{5}$ Normal incorporation results in each pulmonary vein joining the left atrium separately and directly. Faulty incorporation leaves the common pulmonary vein as a distinct structure posteriorly, into which the pulmonary veins empty. This "chamber" is separated from the anterior "fetal" left atrium (containing the left atrial appendage and communicating with the mitral valve) by a diaphragm. This condition, known as cor triatriatum, is one of the rarest of cardiac malformations. ${ }^{6}$ This dividing membrane lies horizontally above the level of the atrial appendage and is composed of cardiac muscle covered on its superior and inferior aspects by endocardial tissue. ${ }^{5}$

Cor triatriatum results if there is minimal incorporation of the common pulmonary vein We believe the abnormality noted on transthoracic exam in these two cases, and identified by transoesophageal study as a prominence at the junction of the left upper pulmonary vein and left atrial appendage, was caused by incomplete incorporation of this membrane into the left atrium. This suggests a continuum between "normal" incorporation of the main pulmonary vein and the syndrome of cor triatriatum. Cardiologists need to be aware of this structure and its possible variants so as to avoid misdiagnosis of this prominence as an atrial tumour or mass.

1 Come PC, Riley MF, Markis JE, Malagold M. Limitations of echocardiographic techniques in evaluation of left atrial masses. Am J Cardiol 1981;48:947-53.

2 Seward JB, Khandheria BK, Oh JK, Abel MD, Hughes RW, Edwards WD, Nichols BA, Freeman WK, Tajik AJ. Transoesophageal echocardiography: technique, anatomic correlations, implementation, and clinical applications. Mayo Clin Proc 1988;63:649-80.

3 Colucci WS, Braunwald E. Primary tumours of the heart. In: Braunwald E, ed. Heart Disease. A Textbook of ED 1988; 1470-83.

4 DePace NL, Soulen RI, Kotler MN, Mintz GS. Two dimensional echocardiographic detection of intraatrial masses. Am J Cardiol 1981;48:954-60.

5 Van Praagh R, Corsini I. Postmortem cases and a study of normal development of the pulmonary vein and atrial septum in 83 human embryos. Am Heart $J$ 1969;78: $379-405$

6 Perloff JK. Congenital mitral stenosis; cor triatriatum; congenital pulmonary vein stenosis. In: The clinical recognition of congenital heart disease. Philadelphia: WB Saunders, 1987; 169-71. 